\title{
SOME QUADRATIC CORRECT EXTENSIONS OF MINIMAL OPERATORS IN BANACH SPACES
}

\author{
I. N. PARASIDIS AND P. C. TSEKREKOS
}

Abstract. Let $A_{0}$ be a minimal operator from a complex Banach space $X$ into $X$ with finite defect, def $A_{0}=m$, and $\widehat{A}$ is a linear correct extension of $A_{0}$. Let $E_{c}\left(A_{0}, \widehat{A}\right)\left(\right.$ resp. $\left.E_{c}\left(A_{0}^{2}, \widehat{A}^{2}\right)\right)$ denote the set of all correct extensions $B$ of $A_{0}$ with domain $D(B)=D(\widehat{A})$ (resp. $B_{1}$ of $A_{0}^{2}$ with $\left.D\left(B_{1}\right)=D\left(\widehat{A}^{2}\right)\right)$ and let $E_{c}^{m}\left(A_{0}, \widehat{A}\right)\left(\operatorname{resp} . E_{c}^{m+k}\left(A_{0}^{2}, \widehat{A}^{2}\right), k \leqslant m, k, m \in \mathbf{N}\right)$ denote the subset of $E_{c}\left(A_{0}, \widehat{A}\right)$ (resp. $E_{c}\left(A_{0}^{2}, \widehat{A}^{2}\right)$ consisting of all $B \in E_{c}\left(A_{0}, \widehat{A}\right) \quad\left(\right.$ resp. $\left.E_{c}\left(A_{0}^{2}, \widehat{A}^{2}\right)\right)$ such that $\operatorname{dim} R(B-\widehat{A})=m\left(\right.$ resp. $\left.\operatorname{dim} R\left(B_{1}-\widehat{A}^{2}\right)=m+k\right)$. In this paper:

1. we characterize the set of all operators $B_{1} \in E_{c}^{m+k}\left(A_{0}^{2}, \widehat{A}^{2}\right)$ with the help of $\widehat{A}$ and some vectors $S$ and $G$ and give the solution of the problem $B_{1} x=f$,

2. we describe the subset $E_{2 c}^{2 m}\left(A_{0}^{2}, \widehat{A}^{2}\right)$ of all operators $B_{2} \in E_{c}^{2 m}\left(A_{0}^{2}, \widehat{A}^{2}\right)$ such that $B_{2}=B^{2}$, where $B$ is an operator of $E_{c}^{m}\left(A_{0}, \widehat{A}\right)$ corresponding to $B_{2}$,

3. we give the solution of problems $B_{2} x=f$.

Mathematics subject classification (2010): 46N20, 47A20, 34B05, 45J05, 45K05.

Keywords and phrases: Minimal operators, correct operators, correct extensions of minimal operators, quadratic correct extensions of minimal operators, solutions of correct problems.

\section{REFERENCES}

[1] R. O. OINARov, I. N. PARASIDI, Correct extensions of operators with finite defect in Banach spases, Izv. Akad. Kaz. SSR, 5 (1988), 42-46 [Russian].

[2] J. Von Neumann, Allgemeine Eigenwerttheorie Hermitescher Functional operatoren, Math. Ann., 102 (1929-1930), 49-131.

[3] V. I. Gorbachuk And M. L. Gorbachuk, Boundary Value Problems for Operator Differential equations, Dordrecht, Kluwer, 1991.

[4] S. G. Krein, Linear equations in Banach spase, Nauka, Moskva, 1971 [Russian].

[5] A. A. DeZIN, General aspects of Boundary Value problems, Moskva "Nauka", 1980 [Russian].

[6] M. I. Vishik, About Boundary Value Problems for Elliptical Differential equations, Mosk. Math. Sosity, 1 (1952), 187-246 [Russian].

[7] E. A. Coddington, Self-adjoint subspace extensions of nondensely defined symmetric operators, Adv. Math., 14 (1974), 309-332.

[8] E. A. Coddington, A. Dijksma, Self-adjoint subspaces and eigenfunction expansions for ordinary differential subspaces, J. Diff. Equat., 20 (1976), 473-526.

[9] E. A. Coddington, A. Dijksma, Adjoint subspaces in Banach spaces with Applications to ordinary differential subspaces, J. Diff. Equat., 20 (1976), 473-526.

[10] A. N. KochUBEI, Extensions of positive definite symmetric operators, D.A.N. USSR, A, 3 (1979), 168-171 [Russian].

[11] A. N. KochUBEI, Extensions of a nondensely defined symmetric operator, Sib. Math. J., 18 (1977), 225-229. 
[12] B. A. MikhaiLets, The spectrum of operators and boundary value problems, spectral analysis of differential operators, Kiev, Inst. of Math. Ukrain. Akadems (1980), 106-131 [Russian].

[13] B. K. Kokebaev, M. Otelbaev, A. N. Shynibekov, About Restrictions and Extensions of operators, D. A. N. SSSR, 271, 6 (1983), 1307-1310 [Russian].

[14] A. N. SHYNIBEKOV, On correct extensions and restrictions for some differential operators, Summary of Ph. D. thesis, Alma-Ata, 1983, 1-16 [Russian].

[15] E. KREYSZIG, Introductory Functional Analysis with Applications, 532. John Wiley and Sons, 1989.

[16] A. N. Kolmogorov, S. V. Fomin, The elements of theory of funtions and functional analysis, "Nauka", Moskva, 1976 [Russian, there is a translation in English]. 